\title{
Saberes profesionales para la transformación del modelo educativo en Chile
}

\author{
Professional knowledge for transforming the educational model in Chile \\ Saberes profissionais para a transformação do modelo educativo no Chile
}

\author{
Nolfa Ibáñez Salgado \\ Universidad Metropolitana de Ciencias de la Educación \\ Correo electrónico: nolfa.ibanez@umce.cl
}

\begin{abstract}
RESUMEN
El presente artículo se fundamenta en resultados del trabajo investigativo que hemos realizado sobre saber pedagógico, discurso y práctica docente en estudios sucesivos en tres regiones del país y en nuestras reflexiones al respecto, en el marco de la situación educativa actual en Chile. Focalizamos nuestra reflexión en el saber pedagógico como entidad epistemológica propia de la profesión docente y en algunos de los aspectos que lo constituyen y que consideramos más relevantes para contribuir al cambio del modelo educativo imperante, entregando propuestas que podrían ayudar a instalar este saber como eje en la formación docente inicial y continua.

Palabras clave: saber pedagógico, formación docente, interacción en el aula.
\end{abstract}

\begin{abstract}
This article is based on results of a research done on pedagogical knowledge, discourse and teaching practice in successive studies in three regions of the country, and our thoughts on the subject in the context of the current educational situation in Chile. We focus our discussion on the pedagogical knowledge as epistemological statute inherent to the teaching profession and some of the aspects that are most relevant and which we consider to help change the prevailing educational model. Some proposals are delivered to help contribute include this knowledge in the teachers' formation.
\end{abstract}

Keywords: pedagogical knowledge, teacher formation, classroom interaction.

\section{RESUMO}

Fundamenta-se em resultados de pesquisa investigativa realizada sobre o saber pedagógico, discurso e prática docente em sucessivos estudos, em três regiões do país e em nossas reflexões sobre o assunto, no contexto da atual situação educacional chilena. Foca-se a discussão sobre o saber pedagógico como essência epistemológica própria da profissão docente e sobre alguns dos aspectos que o constitui e que são considerados mais importantes por contribuírem com a transformação do modelo educacional vigente, entregando propostas que poderiam ajudar na instalação desse saber como eixo da formação inicial e continuada de professores.

Palavras-chave: saber pedagógico, formação docente, interação na aula.

Nos dijeron cuando chicos: "Jueguen a estudiar, los hombres son hermanos y juntos deben trabajar”.

Oías los consejos, los ojos en el profesor,

había tanto sol sobre las cabezas

$Y$ no fue verdad, porque esos juegos al final

terminaron para otros con laureles y futuros,

y dejaron a mis amigos pateando piedras.

Bajo los zapatos: barro más cemento.

\section{El futuro no es ninguno}

de los prometidos en los doce juegos.

A otros enseñaron secretos que a ti no.

A otros dieron de verdad esa cosa llamada educación

Únanse al baile de los que sobran,

nadie los va a echar de más,

¡nadie nos quiso ayudar de verdad!

Extracto de El baile de los que sobran, J. González 


\section{INTRODUCCIÓN}

Resulta poco usual, por decir lo menos, iniciar un artículo académico sobre educación con la letra de la canción de un grupo juvenil de rock que tuvo gran éxito hace más de dos décadas, pero creemos que es útil para la reflexión profunda que debemos hacer sobre las consecuencias que tiene y que debiera tener nuestro quehacer profesional en la vida de las personas que integran la sociedad, a cuyo bienestar la academia a la cual pertenecemos tiene la misión de contribuir. Acercar la academia al mundo de la vida se revela como un gran desafío para las instituciones de educación superior que forman docentes. En esta letra se denuncia el sentir de la mayoría de quienes egresaban a fines de los 80 de una educación pública deteriorada por las decisiones políticas y administrativas tomadas en dictadura; una educación pública que aún no se puede recuperar de aquello, por cuanto las decisiones tomadas con posterioridad a la recuperación de la democracia han conservado la esencia del paradigma educativo instaurado por esa dictadura. El contenido de esta canción anticipó los resultados de numerosas investigaciones sobre la calidad y la equidad de la educación desarrolladas a principios de los 90 en el país, y se mantiene vigente para la realidad que aún viven jóvenes egresados de la educación pública chilena, la cual, como sabemos, ostenta hoy uno de los mayores niveles de segregación del planeta e interpela a muchos para comprender cómo se llega a un sistema 'casi perfecto' de segmentación social de nuestra educación (Concha, 2011).

Si bien el análisis de las causas de esta situación no tiene cabida en el presente trabajo, consideramos importante destacar que el hito que marcó su inicio fue el cambio decretado por la dictadura a principios de la década del 80, que traspasó la administración de los establecimientos educacionales desde el Ministerio de Educación a los municipios a cargo de alcaldes designados por ese mismo gobierno dictatorial, al mismo tiempo que, en el marco de la política neoliberal impuesta en el país, promovió la creación de escuelas por parte de privados, las que podían y pueden hasta hoy recibir dinero del Estado y tener, al mismo tiempo, fines de lucro ${ }^{1}$ (Claude, 2011; Mizala, 2007; Oficina Regional de la Internacional de la Educación para América Latina [ORIEAL], 2003; Paredes y Pinto 2009). En 1980, la matrícula de la educación pública primaria y secundaria abarcaba casi el $80 \%$ de los estudiantes chilenos; hoy, esta se empina apenas por sobre el $40 \%$, siendo Chile uno de los países con mayor segregación escolar a nivel mundial y con insuficientes resultados de aprendizaje en las mediciones nacionales e internacionales (Elacqua, Martínez y Aninat, 2010; Ministerio de Educación [MINEDUC], 2011;Paredes y Pinto 2009; Sistema de Información de Tendencias Educativas en América Latina [SITEAL], 2011). Otros estudios informan que los grados de polarización académica y socioeconómica en la escuela son constantes, en algunos casos crecientes y, lo que es más preocupante, son mayores en los primeros años de escolaridad, lo cual no permitiría seguir considerando al sistema educativo como un espacio para la cohesión social (Villalobos y Valenzuela, 2012). Todo lo anterior se agrava aún más con lo que constatan numerosos estudios en relación a la presencia de discriminación o rechazo a la diversidad en la cultura escolar y en nuestra sociedad en general (Alarcón, 2005; Cerda et al., 2004; Hevia, 2005; Instituto Nacional de Derechos Humanos [INDH], 2011; Magendzo, 2000; Naciones Unidas, 2009; Redon,

Recientemente, en mayo de 2014, ingresó al Congreso Nacional el proyecto de ley que prohíbe el lucro en escuelas y liceos que reciben aportes del Estado. 
Toledo y Rubio, 2009; UNICEF, 2011), por lo que se hace urgente y necesario construir propuestas desde la academia que puedan contribuir a modificar el escenario educativo actual y a hacer posible que se cumplan los propósitos declarados de la profunda reforma educacional que se anuncia en el país.

El presente artículo se fundamenta en reflexiones sobre nuestra educación, a la luz de resultados del trabajo investigativo que hemos realizado sobre saber pedagógico y discurso y práctica docente en estudios sucesivos en tres regiones del país: la Araucanía, Metropolitana y Antofagasta ${ }^{2}$, cuyos principales objetivos fueron: develar concepciones subyacentes a discursos y prácticas docentes en distintos contextos de desempeño y en todos los niveles educacionales, sobre aspectos constitutivos del saber pedagógico; establecer la relación de las prácticas estudiadas en cursos de párvulos y básica con el currículo oficial y algunas de sus recomendaciones, y la relación de esas prácticas con las orientaciones del Programa Intercultural Bilingüe, PEIB, para el caso de los cursos de la Araucanía. En el marco del sistema educacional chileno, focalizamos nuestra reflexión en los aspectos que consideramos más relevantes para contribuir desde nuestro quehacer al cambio de modelo, a la transformación que se requiere en la formación de profesores para que sea posible dicho cambio en el sistema escolar. Estos aspectos forman parte del 'saber pedagógico', entendido como el saber que, en sentido estricto, constituye la especificidad del 'ser' profesor o profesora por cuanto es la entidad epistemológica propia de la profesión docente y sobre la cual debiera desplegarse el contenido de las especialidades y sus didácticas.

En una primera parte, se contextualizará la línea epistemológica y conceptual general de nuestro trabajo. La segunda parte se abocará a lo que entendemos por saber pedagógico y a los principales resultados de la línea de investigación que hemos desarrollado respecto de ese saber, para concluir con algunas propuestas concretas que podrían contribuir a instalar el saber pedagógico como eje en la formación docente inicial y continua, que posibilitará contar con docentes preparados para transformar el modelo educativo actual.

\section{CONTEXTUALIZACIÓN}

En el contexto educacional de los últimos años, nuestro trabajo se ha alineado con el propósito de contribuir a ampliar libertades fundamentales consideradas en las declaraciones y compromisos internacionales sobre desarrollo humano social y económico, como son el acceso equitativo a una educación de calidad y la libertad para intentar lograr aquello que se tiene razones para valorar, así como para salvaguardarlo (Sen, 2007). En Chile, tenemos hoy potentes expresiones ciudadanas que reflejan claramente el intento de hacer valer el derecho a estas libertades fundamentales; entre ellas, están la exigencia por una educación pública gratuita y de calidad, puesta en el centro del debate nacional actual por el movimiento estudiantil secundario y universitario, y la demanda histórica de las personas de los pueblos originarios por la educación y por la preservación y respeto por sus culturas, visibilizada a nivel nacional e internacional principalmente por el pueblo mapuche. Nos hacemos parte de estas dos expresiones, ya que la construcción de la equidad

Línea Saber pedagógico y práctica docente. Investigadora responsable N. Ibáñez: Proyecto FONIDE N²47/2006-2007; Proyecto FONIDE N ${ }^{\circ}$ 55/2008-2009; Proyecto DIUMCE 2008-2010 y 2010/2012. Estudios complementarios a la línea: Tesis postdoctoral Dra. M. Benítez, 2011, UMCE y Proyecto FONDECYT Nº 11090345, investigadora responsable T. Díaz. 
social necesita de la equidad en el sistema educativo, la cual no pasa sólo por la gratuidad y la cobertura, sino particularmente por el encuentro de los futuros ciudadanos en un ámbito que acoja la diversidad y fomente el respeto por el otro diferente; esto es, al alero de un sistema educativo público inclusivo y democrático, desde los niveles iniciales hasta la educación superior.

En la discusión sobre la reforma educacional que ha estado en el tapete, con frecuencia los medios de comunicación masiva consultan a expertos en educación sobre su acuerdo o desacuerdo con los planteamientos gubernamentales y sus opiniones sobre cómo debiese ser llevada a cabo esta reforma. Los expertos son, muchas veces, si no todas, quienes en algún momento han tenido poder de decisión en cuanto a cómo llevar adelante los procesos educacionales en el país, con los resultados por todos conocidos, y no hemos escuchado de ellos y ellas referencias al marco conceptual y epistemológico bajo el cual se llevaron adelante reformas en otras épocas de nuestra historia reciente, cuando no se discutía la calidad de la educación pública. Pareciera que la reflexión sobre y en la educación en Chile se hubiese iniciado recientemente y no existiese la memoria de hitos en nuestra historia educacional en relación a la discusión sobre la modificación del modelo que imperaba en otro momento. En este sentido, y conscientes de que el Chile actual es un país distinto, nos parece relevante rescatar aquí el pensamiento de quienes estaban a cargo de las aulas de escuelas y liceos cuando se discutía otra reforma educacional, y que creemos sería compartido hoy por todos quienes deseamos contribuir a que la educación vuelva a centrarse en el sujeto y en su desarrollo como persona:

Al encarar el problema de la reforma de nuestra educación, procuramos evitar, por lo menos, dos errores en los que generalmente se ha incurrido cuando se han abordado empresas semejantes: 1) el de proceder desde arriba hacia abajo, sin ponerse en contacto con la opinión de los que deben realizar la reforma y de los que deben aceptar sus consecuencias, sin esforzarse por crear un clima dentro del cual vaya produciéndose el cambio de actitudes que, en último término, es lo único que, en verdad, constituye una renovación; y 2) el de creer que una reforma está hecha cuando se han formulado cuidadosamente sus principios teóricos, en general y en detalle, y cuando se les ha sancionado por ley, decreto o reglamento (...), nuestra cultura y nuestra época se orientan hacia la realización de la democracia, es decir, de un régimen de convivencia social que, fundándose en los principios de solidaridad y de respeto de la persona, aspire a constituir una comunidad de hombres libres (...) la función de la escuela es crear un ambiente en el cual los alumnos, mediante la adquisición del patrimonio cultural y la comprensión del mundo en que viven, puedan desarrollar su personalidad (...) lo que consiste, en último término, en la continua transformación del individuo por medio de la experiencia (...) La simple actividad o el simple estudio no constituyen necesariamente una experiencia formativa. Esta existe sólo cuando se establece una relación entre el pensar o el hacer y sus consecuencias sobre la propia vida (...) el dominio de las materias de enseñanza no es, pues, el objetivo final de la educación, sino uno de los medios de encauzar y orientar el desarrollo de la personalidad de los alumnos. En los programas debe acentuarse, más que lo meramente instrumental e informativo, lo formativo y orientador, entendiendo por tal todo aquello que proporcione al alumno la oportunidad de aprender a resolver problemas reales, a conocer, apreciar y valorizar el medio físico y social (...) debe acentuarse, asimismo, la integración de la teoría y de la práctica, base indispensable de un verdadero humanismo, dando lugar, además, a que se manifiesten y satisfagan las diferentes aptitudes e intereses individuales.(...) El contenido de los programas debe destacar no sólo los aspectos intelectuales y técnicos de la cultura, sino también el sentido humano, social y ético con que ésta debe ser empleada; (...) El aprendizaje es un proceso activo del alumno. La tarea del 
profesor consiste en crear un ambiente en el cual este proceso pueda realizarse en las mejores condiciones. (Plan de Renovación de la Educación secundaria Boletín Nº 1, Julio de 1946: 1-4)³.

Coincidiendo con lo expresado a mediados del siglo pasado por educadores dedicados, reflexivos y conscientes de su responsabilidad social en la formación de las niñas, niños y jóvenes chilenos, que tenían claridad respecto al 'para qué' de su educación, y más allá de la esperada concreción de los aspectos administrativos, legales y económicos necesarios para iniciar la reforma que se anuncia en nuestro país, creemos que sólo será posible lograr reconstruir una educación pública de calidad si el sistema cuenta con docentes formados en un saber pedagógico que valore la diversidad, que puedan impulsar la democratización en la institución escolar y operacionalizarla en la implementación del currículo en sus respectivas aulas, de acuerdo a las características de sus contextos de desempeño profesional, lo que conlleva la comprensión de que los estudiantes, con independencia de su edad, origen social, económico o étnico, traen un mundo a la mano que debe ser considerado prioritariamente como base de su proceso de aprendizaje, para lo cual se debe propiciar un estilo relacional en el cual prime una disposición emocional favorable para aprender. Estas competencias profesionales, entendidas como la operacionalización de saberes en un contexto situacional determinado, han sido relevadas por distintos autores, estudios y recomendaciones nacionales e internacionales, reconociendo que la atención pedagógica a la diversidad, la valoración de las experiencias previas y el propiciar un estilo de interacción grato en el aula no sólo mejoraría la calidad de los aprendizajes de los estudiantes, sino también su desarrollo como personas (Cassasus, 2007; Comisión Europea 2002; Ibáñez, 2002; Maturana, 1990; MINEDUC, 2005a; Organización para la Cooperación y el Desarrollo Económico [OCDE], 2010; OCDE-MINEDUC, 2004; Unesco 2000, 2008; entre otros).

Reposicionar una educación pública de calidad, con estatus de derecho social indiscutible, donde se puedan encontrar niños, niñas y jóvenes provenientes de diferentes niveles sociales, culturales y económicos, es un anhelo compartido. Si somos optimistas, podríamos esperar que en el corto y mediano plazo se legisle en una perspectiva distinta al paradigma de mercado que actualmente ostenta el sistema educativo chileno y podamos volver a tener escuelas, liceos y universidades públicos como espacios privilegiados para construir cohesión social, entendida como valores compartidos, donde no prime la competencia por lograr estándares de rendimiento a toda costa y se vuelva a poner el acento en la formación de la persona. Esto implicaría una profunda transformación a nivel de sistema; no pequeños cambios en determinados aspectos, sino un cambio en la concepción misma del hecho educativo y de la proyección que éste tiene para el desarrollo humano de las y los estudiantes; un cambio paradigmático que pueda modificar los modos en que se establecen las relaciones entre los distintos componentes, tanto de diseño, gestión y evaluación, como de materiales y recursos técnicos y, lo más relevante, atender prioritariamente a la formación docente inicial y continua, por cuanto el éxito de cualquier reforma depende de la práctica pedagógica cotidiana de las profesoras y profesores que tienen a su cargo la convivencia con niños, niñas y jóvenes en la escuela y el liceo. Esta transformación no es fácil ni rápida, porque sabemos que los cambios paradigmáticos toman tiempo y que quienes actúan bajo paradigmas distintos "son como los miembros de

Documento original facilitado a la autora en 1994 por el gran educador Hernán Vera Lamperein, integrante del equipo que lo elaboró, quien recibiera el Premio Nacional de Educación en 2001, pocos años antes de su desaparición. 
comunidades de distinta cultura-lenguaje" (Kuhn, 1980: 312); los significados y sentidos, así como las prioridades, se encauzan por distintos caminos; unos 'no ven' lo que 'ven' los otros y viceversa; no obstante y al menos en el discurso, la formación docente en Chile declara paradigmas de formación que se comparten en lo sustantivo, por lo que la gran tarea sería hacer coherente la práctica pedagógica con dichos paradigmas. El profesor o profesora, como ningún otro profesional, tiene a su cargo un espacio de convivencia prolongada con sus estudiantes, y por tanto una gran responsabilidad en el curso que toman las percepciones y creencias sobre el mundo de la vida que están construyendo esos niños, niñas y jóvenes. Por esta razón, sostenemos que el saber pedagógico, entendido como la especificidad del 'ser' docente, debiese ser el eje que estructure un camino posible hacia la disminución de la polarización académica y socioeconómica existente en nuestro sistema escolar, y, al mismo tiempo, hacia la visibilización de la riqueza que implica hacerse cargo de la diversidad de construcciones de mundo que traen los estudiantes a la sala de clase.

\section{SABER PEDAGÓGICO}

El término saber pedagógico refiere a los conocimientos y competencias -entendidos como elementos relacionales, procesuales, situados y dinámicos- que constituyen los fundamentos para la acción pedagógica que se realiza al interior de la institución educativa, con la intencionalidad que la sociedad le otorga en cada época. No existe una conceptualización compartida sobre este saber en la formación docente (Abraham, 2009; Ibáñez, 2010; Nervi y Nervi, 2007; Restrepo, 2004; Villata, 2004); como indica Zapata "el campo del saber pedagógico no está organizado homogéneamente y, además, sus bordes no están claramente delimitados" (2003: 180). Lo anterior dificulta la priorización en las competencias que serían específicas del 'saber enseñar', más allá del necesario manejo del contenido disciplinar y de su didáctica. Tomamos como base la conceptualización que recoge el Informe de la Comisión sobre Formación Inicial Docente (MINEDUC, 2005a ), a partir de la revisión de estudios nacionales e internacionales y de la consulta a expertos, que señala que los principales contenidos o saberes constitutivos del saber pedagógico son: “ a) el saber sobre cómo ocurre el aprendizaje, qué lo facilita y qué lo dificulta; $b$ ) el saber sobre el contexto histórico y general del grupo de estudiantes, de la escuela y su currículo; c) el saber sobre cómo facilitar un contexto interaccional adecuado en cada aula (...); y d) el conocimiento y comprensión del contenido de la especialidad y de su didáctica" (2005a: 58). A lo anterior, agregamos el saber sobre la atención pedagógica a la diversidad propia de cada grupo curso.

Nuestra concepción de 'saber pedagógico' distingue a este saber como un corpus de conocimientos provenientes de distintas disciplinas, articulado por la comprensión de la relación dialógica entre teoría y práctica, cuyo nodo central es el entendimiento sobre cómo ocurre el aprendizaje, qué lo facilita y qué lo dificulta (Ibáñez, 2007). A partir de este conjunto de saberes articulado por la comprensión que el profesor o profesora tiene de la relación teoría-práctica en su contexto de desempeño, él o ella desplegará su conocimiento del contenido a enseñar y propiciará en su clase un determinado contexto interaccional, una orientación particular de las relaciones interpersonales al interior del aula. El saber pedagógico es sistémico, y por su carácter situado es siempre dinámico, por lo que aparece incomprensible a la mirada lineal. A pesar de sus implicancias en las políticas públicas de educación y en los programas formativos, este saber cuyas bases debieran construirse 
tempranamente en el proceso formativo de los futuros docentes, ha sido poco estudiado sistemáticamente (Díaz, 2005; Mercado, 2002; Nervi, 2006). Al respecto, Nervi y Nervi (2007) hacen una revisión de la concepción científica de la pedagogía asumida por distintos autores y concluyen: "Hay consenso entre los teóricos, especialmente europeos, en que el objeto de la pedagogía sería la relación educativa con propósitos de formación humana, y ello implica conceptuar a ese objeto como entidad específica en toda su complejidad empírica" (2007: 47). La pedagogía por tanto no es neutra o inocente (Tardiff, 2005) y el saber pedagógico se construye y reconstruye en un sistema educativo institucionalizado, escolar o no, que, como se dijo antes, intenciona la formación humana en la perspectiva que la sociedad adopta en cada época. La intencionalidad presente en el discurso sobre la reforma educativa que se anuncia hoy, cuyo fin es el término de la segregación y que concita la aprobación de la mayoría de la sociedad chilena, requiere de un saber pedagógico centrado en la persona del estudiante, en su visión de mundo y en la valoración de su diversidad en cuanto conocimientos, habilidades y actitudes.

El saber pedagógico así entendido, requiere por tanto de la complementariedad entre el conocimiento experto y el conocimiento situado del cotidiano escolar, de la capacidad de operacionalizar o poner en acto en la situación de aula aquello en lo que se cree. Tanto en Chile como en otros países latinoamericanos, la investigación disponible muestra que no existiría una cabal comprensión de la relación entre la teoría que se aprende en la formación o en el perfeccionamiento y la práctica pedagógica en situaciones concretas de aprendizaje-enseñanza. Las prácticas constituirían "el núcleo más difícil de cambiar de los sistemas educativos (...), éstas todavía exhiben mayor desconexión con los requerimientos de la profesión en contextos de desempeño real" (Cox, 2007:14), esto es, entre la formación y la vida. En general, las prácticas docentes se enmarcan en la lógica dual de la reacción-reproducción (Latorre, 2003) y persiste la falta de coherencia entre el discurso pedagógico aceptado por las/los docentes y sus prácticas en el aula, incluidos los docentes formadores (Alegría y Cisternas, 2003; Cabrera, 2003; Carrasco y Montes, 2003; Chávez, 2007; Cisternas, 2012a; Ibáñez et al., 2009; Jáuregui y Miller, 2002; Valenzuela, 2006; Vaillant, 2004), lo que parece constituir uno de los mayores obstáculos epistemológicos para el mejoramiento de la educación y que estaría a la base de la débil asociación entre los procesos de formación inicial docente y el desempeño profesional en el aula (Flórez, 1994; MINEDUC, 2005a; Ortuzar et al., 2009). Lo anterior estaría indicando que en la formación de profesores "hay ciertas prácticas y modelos de formación que no van más allá de un conocimiento 'declarativo' y obstaculizan el aprendizaje situado de las prácticas de enseñanza” (Cisternas, 2012b: 8).

La práctica del profesor o profesora conlleva, por cierto, los principios pedagógicos y científicos que él o ella acepta, pero al mismo tiempo conlleva también sus preferencias, percepciones estéticas, juicios éticos y visiones políticas, sociales y culturales, todo lo cual permea su estilo de relación con los estudiantes y orienta distintos modos de convivencia, según se privilegie o no la participación, el respeto por la opinión distinta y la valoración de las experiencias previas (Cornejo y Redondo, 2001; Díaz, T., 2007; Díaz, V., 2005, 2010; Ibáñez et al., 2009). En cuanto al estilo de interacción, este puede favorecer u obstaculizar el rol que los estudiantes debieran tener en la toma de decisiones del docente sobre cómo enseñar determinados contenidos, especialmente en contextos donde la cultura del estudiantado implica un "habitus" particular, distinto al de la cultura dominante (Díaz y Druker, 2007; Sánchez, 2003). Como señala von Bertalanffy: 
Somos nosotros [los profesores] quienes verdaderamente fabricamos las gafas a través de cuyos cristales la gente ve el mundo y se ve a sí misma, sin saber y sin darse cuenta de quienes fueron los que se las calaron sobre su metafórica o metafísica nariz. Es posible que seamos los grandes fabricantes de gafas de la historia. Por eso, la labor intelectual es algo más que acopiar datos o construir ingeniosos artefactos. Es una responsabilidad tremenda y debemos afrontarla. (1974: 76).

Dado que la formación y el perfeccionamiento docente se basan en teorías de tipo socio constructivistas que son ampliamente aceptadas y bajo el supuesto de que todos los profesores y profesoras, de cualquier nivel educacional, desean que sus estudiantes aprendan aquello que se proponen como objetivos de aprendizaje, entonces podemos inferir que a la base de este gran problema de poca coherencia entre lo que se dice y lo que se hace estaría, principalmente, la dificultad de los formadores para operacionalizar en el aula los marcos conceptuales y epistemológicos sobre aprendizaje que han adoptado desde la teoría, para poner en acto aquello que conceptualmente comprenden y aceptan. Dicho de otro modo, y como ha sido señalado antes (Schiefelbein, 1993), si los estudiantes que se están formando como docentes no tienen, en general, la posibilidad de tener la experiencia cotidiana de modos de implementar el currículo que sean coherentes con las teorías que están aprendiendo, se seguirán replicando los modos de enseñanza tradicionales aun cuando correspondan a concepciones de aprendizaje ya dejadas de lado en la formación.

Un aspecto positivo que permite visualizar un punto de partida para el cambio, es que sabemos que existe consenso entre formadores y expertos sobre la necesidad de introducir modificaciones importantes en la formación docente (Avalos, 2003, en línea; Avalos y Matus, 2011; Cornejo y Redondo, 2007; MINEDUC 2005a; Síntesis conclusiones $2^{\circ}$ Encuentro Nacional de Formación Docente: UCT, 2006; Síntesis conclusiones $3^{\circ}$ Encuentro Nacional de Formación Docente UCSH, 2007; Síntesis conclusiones y $4^{\circ}$ Encuentro Nacional de Formación Docente UCV y UPLACED, 2008; Suárez, 2006), y podríamos esperar, entonces, un ambiente propicio para la transformación en las instituciones de educación superior que forman profesores, en el marco de la reforma a la educación que hoy se discute, puesto que "cualquier intento o proyecto de cambio e innovación suele comenzar y adquirir cierta proyección y trascendencia gracias a la toma de conciencia, al esfuerzo y a los aportes de individuos o pequeños grupos de éstos convencidos y motivados por la necesidad del cambio y su orientación de acuerdo a un sentido determinado" (Cornejo y Redondo, 2007: 52). Si la intención de la educación chilena en su conjunto es orientar la formación humana hacia una convivencia democrática en la cual la diversidad sea vista como un valor, entonces la formación docente tiene un rol protagónico en la contribución efectiva a la ampliación de las libertades fundamentales de las y los estudiantes y sus familias al impulsar, como dijeron los educadores del siglo pasado citados antes, "un régimen de convivencia social que, fundándose en los principios de solidaridad y de respeto de la persona, aspire a constituir una comunidad de hombres libres" (Cornejo y Redondo, 2007: 52).

\section{ALGUNOS RESULTADOS DE LA LÍNEA DE INVESTIGACIÓN}

Las investigaciones de la línea sobre saber pedagógico y discurso y práctica docente, que tuvo los objetivos ya señalados, consistió en estudios cualitativos comparativos del análisis de clases observadas en 12 aulas, desde párvulos a $6^{\circ}$ básico, de 8 escuelas de 4 
comunas de Santiago; en 11 aulas de los mismos niveles, completas y multigrado, de 5 escuelas rurales de comunidades mapuche de 3 comunas de la Araucanía (Ibáñez et al., 2009); y en 12 cursos desde párvulos a $7^{\circ}$ básico de 2 establecimientos educativos de la ciudad de Antofagasta y uno de San Pedro de Atacama (Benítez, 2011). Todos estos estudios fueron realizados con idéntica metodología, y a ellos se suman dos estudios complementarios: uno que indaga sobre el saber pedagógico y la formación inicial docente en la práctica pedagógica en contextos de educación intercultural en la Región de la Araucanía, desde la perspectiva de la experiencia de docentes mapuche en ejercicio (Díaz, 2012); y el otro que analiza discursos de 51 formadores de docentes pertenecientes a 14 Departamentos Académicos de la UMCE y de 75 profesoras y profesores de 7 escuelas y liceos de dos comunas de Santiago, en relación a algunos aspectos del saber pedagógico ${ }^{4}$ (Ibáñez, 2012). Estos estudios se desarrollaron entre 2006 y 2012.

Los aspectos constitutivos del saber pedagógico estudiados son: el saber sobre cómo ocurre el aprendizaje, el saber sobre cómo facilitar un contexto interaccional en el aula que lo favorezca, y el saber sobre la atención pedagógica a la diversidad. Para establecer la caracterización de la concepción general de aprendizaje que como observadores podíamos distinguir, se consideraron dos perspectivas generales: a) la que supone que el/ la docente determina lo que el estudiante aprende, que corresponde a las explicaciones o teorías de tipo asociacionistas que asumen que el medio especifica lo que ocurre en el ser humano, y que fue el paradigma predominante en la formación de profesores por mucho tiempo; y b) la que supone que el aprendizaje es un proceso activo de construcción desde el propio estudiante y que corresponde a las explicaciones o teorías de orientación socio constructivista que constituyen el paradigma declarado hoy en día en la formación docente, y que asumen que el nuevo aprendizaje se construye basado en conocimientos y experiencias previas, lo que implica atender al contexto de vida del estudiante, a sus intereses y emociones. El marco teórico de esta línea de investigación, además de lo que aquí se ha señalado, consideró la revisión de autores como T. da Silva, H. Maturana, R. Kane, S. L. Shulman, M.A. Sánchez, D. Shön, J. Tardif y von Foerster, entre otros, así como la producción anterior de los integrantes del equipo de investigación.

En estos estudios no se pretendía sólo conocer el discurso de los docentes sobre las teorías o concepciones a que adscriben sino que, a través de analizar sus acciones y explicaciones respecto de estas, develar la concepción general de aprendizaje subyacente a su quehacer profesional y sus valoraciones en relación a los otros aspectos del saber pedagógico estudiados. Para las clases analizadas desde párvulos a $7^{\circ}$ básico, se utilizaron videograbaciones de, al menos, tres clases consecutivas, las cuales se revisaron posteriormente en conjunto con el o la docente, focalizando el análisis en el contexto interaccional que se propicia, el modo en que es acogida, o no, la diversidad de los estudiantes, la relación de la práctica tanto con el currículo oficial como con los criterios y descriptores del Dominio B del Marco para la Buena Enseñanza, MBE, (MINEDUC, 2003) y, en el caso de los cursos de escuelas rurales de la Araucanía, con las orientaciones y propósitos del Programa de Educación Intercultural Bilingüe, PEIB (MINEDUC, 2005b). Para el caso de los formadores y de los profesores del sistema de quienes se recogió sólo su discurso, previamente todos habían participado en la evaluación del contexto interaccional en sus respectivos cursos o carreras ${ }^{5}$, por lo

Proyecto DIUMCE 2010/2011.

Línea de Investigación: Las emociones en el aula: Proyectos DIUMCE 2000-2008. 
que la pretensión fue visibilizar la concepción de aprendizaje que declaraban aceptar y la importancia que daban a los otros aspectos del saber pedagógico considerados en el estudio.

Los resultados mostraron que, en los discursos de la mayoría de los docentes formadores y del sistema, se refleja una concepción general de aprendizaje más cercana a las de tipo socio constructivista. En su gran mayoría, ellos expresan la importancia de tomar en cuenta las experiencias previas del estudiante, de propiciar un clima grato para facilitar el aprendizaje y reconocen la relación emociones-aprendizaje, evidenciando autocrítica al respecto y auto proponiéndose modos de mejorar en tal sentido la propia práctica (Ibáñez, 2011). No obstante, y especialmente en el caso de los formadores, la aceptación de la diversidad del estudiantado se encuentra prácticamente ausente, y cuando lo expresado refiere a este aspecto, mayormente se hace la referencia en un sentido negativo. En la mayoría de los cursos cuyas interacciones se analizaron, estas se enmarcan en premisas de pensamiento que corresponden a una concepción general de aprendizaje que no se considera en la formación desde hace décadas. Sin embargo, sus profesoras y profesores declaran adscribir a una concepción de aprendizaje actual de tipo socio-constructivista y están genuinamente interesados en aportar al mejoramiento educacional, como lo demuestra su comprometida colaboración. Esto constataría la no existencia de una comprensión cabal de la relación entre la teoría que se aprende y la práctica en situaciones concretas, que es lo que articula los saberes constitutivos del saber pedagógico.

Los aspectos del saber pedagógico considerados para el análisis en estos estudios, son operacionalizados en las prácticas estudiadas de distintos modos, según sea la concepción de aprendizaje subyacente al quehacer profesional del profesor o profesora y el contexto interaccional que él o ella propicia en el aula, lo que estaría indicando que estos son dos dominios fundamentales a considerar en la formación docente inicial y continua. Los/las docentes operacionalizan su quehacer de modo consistente con las premisas de pensamiento subyacentes: si piensan que lo externo es lo que informa de su existencia, entonces enfatizan la repetición, no favorecen distintos modos de ejecución, priorizan el trabajo individual y las evaluaciones con preguntas de respuesta única, porque el objetivo es que los conceptos, las relaciones y los objetos se aprendan tal como son, objetivamente. En cambio, si la concepción subyacente supone que el aprendizaje es un proceso que se determina desde el estudiante, a partir de sus distinciones, donde los cambios que en él ocurren se sustentan en su bagaje experiencial, emocional y cognoscitivo, entonces los docentes, además de propiciar la participación, contextualizan los contenidos en el mundo cotidiano de sus estudiantes, enfatizan el trabajo colaborativo, valoran las experiencias e incorporan la diversidad de sentidos y significados presentes en el grupo a la temática de la clase, todo lo cual implicaría el aprendizaje significativo de los nuevos conceptos, relaciones u objetos. Esta concepción de aprendizaje es muy minoritaria en las clases analizadas.

La mayoría de los profesores y profesoras cuyas clases se estudiaron implementan el currículo de modo similar, con independencia de las tremendas diferencias entre sus contextos de desempeño. Constatamos que el propiciar un contexto interaccional grato para los estudiantes no se vincula necesariamente con una concepción de aprendizaje determinada y sí con las creencias del docente sobre la imbricación entre aprendizaje y emociones. Al comparar las aulas observadas, la mayor diferencia la constituye el comportamiento de los estudiantes de aulas rurales de la Araucanía, donde ellos y ellas se muestran siempre interesados en la realización de las actividades y no se observa ninguna conducta que pudiese considerarse disruptiva o de desagrado hacia los pares, profesores y/o 
hacia la situación de aprendizaje. La mayoría de las/los docentes implementa el currículo oficial sin modificaciones, sin integrar distintos subsectores de aprendizaje ni realizar adecuaciones curriculares; no obstante, la presencia de estos aspectos, aunque escasa, se visualiza en mayor medida en las aulas rurales de la Araucanía. Del mismo modo, la correspondencia con el Dominio B del MBE es mayor en las prácticas docentes observadas en las escuelas rurales de esa región. Respecto al PEIB y con sólo con dos excepciones, en las prácticas analizadas no se reflejan sus orientaciones, aun cuando la mayoría o la totalidad del estudiantado es mapuche y algunos docentes han sido capacitados por ese programa. Esto es, no se advierten contenidos contextualizados en el mundo cotidiano de los estudiantes ni un enfoque intercultural entendido como diálogo entre culturas.

El resultado que más nos interpela es la constatación de que la aceptación e incorporación de la diversidad de construcciones de mundo -sentidos y significados- que trae el estudiantado a los contenidos o temática de las clases, es la competencia profesional docente con menor presencia en las prácticas estudiadas, lo que es consistente con los resultados del análisis del discurso de formadores y profesores del sistema. Esto, no obstante la preeminencia otorgada a este aspecto en el discurso educativo, tanto en el de las políticas públicas como en el de las instituciones formadoras. Es urgente, entonces, implementar cambios en la formación inicial y continua que posibiliten al formador, al futuro docente y al docente en ejercicio tener la experiencia, en el proceso de construcción y reconstrucción de su saber pedagógico, de ser sujeto de aplicación de la concepción de aprendizaje que se acepta en la teoría y que implica hacerse cargo pedagógicamente de la diversidad presente en cada aula, para contribuir así, desde la academia, a la equidad educativa de nuestro sistema escolar, que es condición necesaria para lograr la equidad social.

\section{REFLEXIONES FINALES Y PROPUESTA}

Pensamos que para lograr un cambio sustantivo en la práctica pedagógica que posibilite el tránsito hacia la educación que anhelamos, es necesario que los formadores, los docentes del sistema escolar y los estudiantes de pedagogía "se apropien" de lo que aceptan en el discurso como concepción de aprendizaje y aprendan a trabajar pedagógicamente con la diversidad. Es evidente la necesidad de una rearticulación de la teoría-práctica en los procesos formativos que permita la vivencia de la relación dialógica entre lo que el estudiante y el docente aprenden en su formación y/o perfeccionamiento sobre el aprendizaje y la interacción humana, y el "aterrizaje" de aquello en su propia experiencia como estudiante o profesor-estudiante. Dado que las teorías declaradas como fundamento de la formación y el perfeccionamiento docente son ampliamente compartidas, podemos inferir que el principal obstáculo epistemológico para la transformación de la práctica pedagógica en la escuela no es la comprensión de la teoría como tal, sino la comprensión de la relación dialógica entre esa teoría y la práctica concreta en contextos particulares, cuestión de la que debe hacerse cargo la formación docente. Por esto, reiteramos la recomendación planteada ya antes a las instancias ministeriales a partir de las evidencias recogidas en algunos de los estudios aquí mencionados ${ }^{6}$ : considerar estos aspectos en el perfeccionamiento docente, en los programas o cursos de las distintas áreas y niveles, enfatizando el rol del docente

Recomendaciones para las Políticas Públicas. Informes Finales proyectos FONIDE N 247 y 55. 
como profesional de la disciplina pedagógica cuyo objeto de estudio es la educación para el desarrollo humano de quienes son sus estudiantes.

La priorización del rol técnico de los profesores y profesoras, quienes cada vez más se esfuerzan por seguir las indicaciones y sugerencias del currículo oficial para que sus estudiantes alcancen mejores resultados en las evaluaciones nacionales, oculta la base necesaria de entendimiento provisto por su formación, que es lo que le permitiría recrear nuevas estrategias y metodologías basadas en las concepciones de aprendizaje que aprendieron en teoría, pero que casi no tuvieron ocasión de vivenciar como estudiantes. La formación docente inicial y el perfeccionamiento de los profesores en servicio serían las instancias a través de las cuales se podría propiciar un cambio efectivo en las premisas de pensamiento de los docentes acerca de cómo ocurre el aprendizaje en sus estudiantes y poder desarrollar competencias profesionales para el trabajo pedagógico con la diversidad del estudiantado, diversidad que es condición de la situación educativa y cuyo abordaje permitiría disminuir la inequidad de nuestra escuela. Las experiencias de formación que le permitan tener al estudiante y al profesor-estudiante la vivencia de modos de interacción en la situación de aprendizaje que sean coherentes con las teorías que aprende, podría ser una manera eficaz de instalar como eje el saber pedagógico que constituye la especificidad de la profesión docente, propiciando que el cambio de paradigma fuese efectivo en la práctica y no solo compartido en el discurso.

La nuestra es una propuesta concreta, no teórica: si los cursos de los programas de formación docente y continua, en cualquier disciplina y para cualquier nivel, consideraran una unidad de correspondencia teoría-práctica en la cual el formador explicitara o mostrara a sus estudiantes y profesores-estudiantes la coherencia entre la teoría de aprendizaje en la que se basa (o que enseña y/o acepta) y el modo en que estructuran las situaciones de aprendizaje y de evaluación en ese curso en particular, podría iniciarse un camino hacia la resolución del problema que hemos analizado en este trabajo y configurarse una conceptualización compartida del saber pedagógico en la formación de profesores. Como la profesión docente significa también períodos prolongados de convivencia con niñas, niños y jóvenes, hay que hacerse cargo de la interacción profesor-alumno, que tiene un carácter formativo y, como se dijo antes, incide en el desarrollo humano de los estudiantes, lo que hace que la responsabilidad del profesor o profesora se extienda más allá de la enseñanza de las disciplinas y del logro de determinados estándares de rendimiento, especialmente en los primeros años de escolaridad. Los niños y niñas van configurando sentidos y significados para objetos, conceptos y relaciones dependiendo del modo en que estos tienen presencia en sus vidas, y esos sentidos y significados formarán parte de su visión de mundo; esto es, la responsabilidad para con las propias acciones, la coherencia entre lo que se dice y lo que se hace, el ejercicio democrático, el respeto por el otro diferente y la solidaridad, por nombrar sólo algunos aspectos, no se aprenden de textos ni de la exposición oral de un profesor, sino que se aprenden según el modo en que se viva, se aprende en la convivencia. Esto debiese ser una consideración central en la formación docente.

Esperamos con optimismo que la reforma que se anuncia sea, en verdad, un cambio estructural que transforme el enfoque de mercado que hoy impera en la educación chilena por un enfoque de derechos que permita disminuir la inequidad de la que nos habla la letra de la canción con la que iniciamos este artículo, y que cala más hondo que los datos estadísticos, y posibilitar que nuestra educación cumpla, finalmente, la promesa que su discurso hace a los niños, niñas y jóvenes chilenos y que está pendiente desde hace mucho. 
Creemos que esto es posible, en la medida en que quienes tenemos a cargo la formación de profesores tengamos también la disposición a soltar nuestras certezas y mirarlas, para reflexionar sobre ellas y decidir si queremos conservarlas o innovar.

\section{REFERENCIAS BIBLIOGRÁFICAS}

Abraham, M. (2009). Pedagogía, saber pedagógico y prácticas educativas. Paulo Freire. Revista de Pedagogía Crítica, año 8, n.7, 119-131.

Alarcón, P. (2005). Hacia una pedagogía de la coexistencia. Paulo Freire. Revista de Pedagogía Crítica, n.3, 63-84.

Alegría, M. y Cisternas, T. (2003). Significados y Saberes que Orientan las Acciones Educativas de Estudiantes de Pedagogía de la Universidad Metropolitana de Ciencias de la Educación. Actas Congreso FID 2003, UMCE, Santiago.

Ávalos, B. (2003). La formación docente inicial en Chile, Santiago. Digital Observatory for Higher Education in Latin America and the Caribbean. Recuperado desde http://www.oei.es/ docentes/info_pais/informe_formacion_docente_chile_iesalc.pdf

Ávalos, B. y Matus, C. (2011). La formación Inicial Docente en Chile desde una óptica internacional. Informe nacional del Estudio Internacional IEA TEDS-M (2012). Santiago: MINEDUC.

Benítez, M. (2011). Saber pedagógico y prácticas docentes en contexto rural atacameño y urbano en escuelas de la II Región de Antofagasta (Tesis Postdoctoral). UMCE, Santiago de Chile.

Cabrera, J. (2003). Discurso docente en el aula. Estudios Pedagógicos, n.29, 7-26.

Casassus, J. (2007). La educación del ser emocional. Santiago: Cuarto propio.

Cerda, A., Egaña, M., Magendzo, A., Santa Cruz, E. y Varas, R. (2004). El complejo camino de la formación ciudadana. Santiago: PIIE.

Cisternas, T. (2012a). Contextos, escenarios y disposiciones en el origen de las investigaciones sobre Formación Docente realizadas en Chile. Un Estudio de casos. Ponencia presentada al Segundo Congreso Interdisciplinario de Investigación en Educación. CIIE 2012. Recuperado desde http:// www.ciie2012.cl/?page=view_programa_completo

Cisternas, T. (2012b). Dificultades experimentadas por profesores principiantes de educación básica en sus prácticas de enseñanza y su relación con la formación inicial. Ponencia presentada al Segundo Congreso Interdisciplinario de Investigación en Educación. CIIE 2012. Recuperado desde http://www.ciie2012.cl/?page=view_programa_completo

Claude, M. (2011). La educación pública es posible. Santiago: Universidad de Chile. Recuperado desde www.archivochile.com/Chile_actual/01_mse/5/MSE5_0016.pdf

Comisión Europea. (2002). Informe Comisión Europea. Educación y formación en Europa: sistemas diversos, objetivos compartidos para 2010. Dirección General de Educación. Recuperado de http://www.peretarres.org/eutses/recursos/educacion.pdf

Comisión Nacional de Formación Docente. (2006). Síntesis conclusiones $2^{\circ}$ Encuentro Nacional sobre Políticas de Formación Docente. Universidad Católica de Temuco.

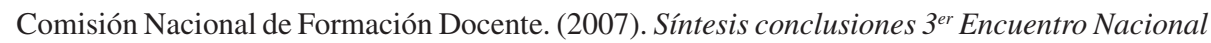
sobre Políticas de Formación Docente. Universidad Católica Silva Henríquez.

Comisión Nacional de Formación Docente (2008). Síntesis conclusiones $4^{\circ}$ Encuentro Nacional sobre Politicas de Formación Docente. Universidad de Playa Ancha, Facultad de Ciencias de la Educación. Universidad Católica de Valparaíso.

Concha, C. (2011). Chile, cerrando el ciclo de la administración educativa municipal. Ponencia presentada en el Seminario Regional Descentralización de la Educación en Centroamérica: Mejora de la Eficiencia y la Participación. GIZ, ICAP y Ministerio de Educación de Guatemala. Ciudad de 
Guatemala. Recuperado de http://biblioteca.uahurtado.cl/ujah/reduc/pdf/pdf/txt800.pdf

Cornejo, J. (2007). La formación de los formadores de profesores: ¿Para cuándo en Chile?

Pensamiento Educativo, vol.41, n.2, 37-55.

Cornejo, R. y Redondo, J. (2001). La importancia de los factores socio ambientales e interpersonales al interior de las instituciones escolares. Última Década, n.15, 11-52.

Cornejo, R. y Redondo, J. (2007). Variables y factores asociados al aprendizaje escolar. Una discusión desde la investigación actual. Estudios Pedagógicos, vol.33, n.2, 155-175.

Cox, C. (2007). Educación en el Bicentenario: dos agendas y calidad de la política. Pensamiento Educativo, vol.40, n.1, 175-204.

Chaves, E. (2007). Inconsistencia entre los programas de estudio y la realidad de aula en la enseñanza de la estadística de secundaria. Actualidades Investigativas en educación, vol.7, n.3, 1-35.

Díaz, V. (2005). Teoría emergente en la construcción del saber pedagógico. Revista Iberoamericana de Educación, n.37/3, 1-18. Recuperado desde http://www.rieoei.org/1122.htm

Díaz, V. (2010). Fundamentos teóricos del saber pedagógico. Investigación y Postgrado. vol.25, n.2-3,98-108. Recuperado desdehttp://www.scielo.org.ve/scielo.php?script=sci_arttext\&pid=S131600872010000200009\&lng=es\&nrm=iso

Díaz, T. (2007). Las relaciones pedagógicas profesor estudiante como configuradoras de escenarios curriculares intra aula (Tesis Doctoral). Pontificia Universidad Católica de Chile, Santiago de Chile.

Díaz, T. (2012). Informe Final Proyecto FONDECYT N 11090345.

Díaz,T. y Druker, S. (2007). La democratización del espacio escolar: Una construcción en y para la diversidad. Estudios Pedagógicos, vol.33, n.1, 63-77.

Flórez, R. (1994). Hacia una pedagogía del conocimiento. Caracas: McGraw Hill.

Elacqua, G., Martínez, M. y Aninat, C. (2010). ¿Cómo fortalecer la Educación Municipal? Capacidad y responsabilidad política. En S. Martinic y G. Elacqua (Eds.), ¿Fin del Ciclo? Cambios en la Gobernanza del Sistema Educativo (pp. 101-130). Santiago: Unesco. Pontificia Universidad Católica de Chile.

Hevia, R. (2005). Culturas originarias: Integración y desarrollo de nuestra diversidad. En Consejo Nacional de la Cultura y las Artes. En Diversidad Cultural. El valor de la Diferencia (pp. 99-112). Santiago: LOM Ediciones.

Ibáñez, N. (2002). Las emociones en el aula. Estudios Pedagógicos, n.28, 31-45.

Ibáñez, N. (2007). Informe Avance Proyecto FONIDE No 274 (ex 8513).

Ibáñez, N. (2010). La atención pedagógica a la diversidad. Estudio en aulas de escuelas rurales de comunidades mapuche del sur de Chile. Educación Superior y Sociedad, año 15, n.2, 83-110.

Ibáñez, N. (2011). Las emociones de los estudiantes: factor relevante en la construcción y reconstrucción del saber pedagógico. Novedades Educativas, n.251, 10-16.

Ibáñez, N. (2012). Saber pedagógico: su reconstrucción en el discurso de docentes formadores de profesores y del sistema escolar. Informe final Investigación. Proyecto DIUMCE. Universidad Metropolitana de Ciencias de la Educación.

Ibáñez, N., Díaz, T., Druker, S. y Rodríguez, M. (2009). Saber pedagógico y práctica docente: Estudio en aulas de educación parvularia y básica. En Selección de Investigaciones Primer Concurso FONIDE: Evidencias para Políticas Públicas en Educación (pp. 55-97). Santiago: MINEDUC.

Instituto Nacional de Derechos Humanos. (2011). Primera Encuesta Nacional de Derechos Humanos. Autor. Recuperado desde www.indh.cl

Jáuregui, M., Carrasco, L. y Montes, I. (2003). Evaluando, evaluando: ¿Qué piensa y qué hace el docente en el aula? Informe Final de Investigación. Universidad Católica Santa María. Perú. Recuperado desde http://cies.org.pe/files/active/0/R0204.pdf

Latorre, M. (2003). En el ojo del huracán: ¡qué características tiene el "Saber pedagógico en uso" de nuestros profesores? ¿Es posible contribuir desde la formación inicial? Boletín de Investigación Educacional, vol.18, 285-296. 
Magendzo, A. (2000). La diversidad y la no discriminación: Un desafío para una educación moderna. Pensamiento Educativo, vol.26, 173-200.

Maturana, H. (1990). Emociones y lenguaje en educación y política. Santiago: Hachette.

Mercado, R. (2002). Los saberes docentes como construcción social. México: Fondo de Cultura Económica.

Miller, E. (2002). Políticas de formación docente en la mancomunidad del Caribe. En UNESCO/ OREALC, Formación Docente: un aporte a la discusión (pp. 15-34). Unesco/Orealc.

MINEDUC. (2003). Marco para la Buena Enseñanza. Santiago: Autor.

MINEDUC. (2005a). Informe de la Comisión sobre Formación Docente Inicial. Santiago: MINEDUC, Serie Bicentenario.

MINEDUC. (2005b). Orientaciones para la Contextualización de Planes y Programas para la Educación Intercultural Bilingüe. Santiago: Autor.

MINEDUC. (2011). Informe PISA 2009. Recuperado desde www.simce.cl/index. php?id=100\&no_cache $=1$

Mizala, A. (2007). La economía política de la reforma educacional en Chile. Serie Estudios Socio Económicos, n.36. Santiago de Chile: CIEPLAN. Recuperado desde www.cieplan.org/media/.../153/ Capitulo_1.pdf

Naciones Unidas. (2009). Informe Naciones Unidas Examen Informe CERD Chile. Agosto 2009. Recuperado desde http://www.politicaspublicas.net/docs/2009_08_cedr_obs_chile.pdf

Nervi, M. (2006). El saber pedagógico en Chile: Un contexto Contradictorio. Perspectivas Educacionales, n.6, 25-33.

Nervi, M. y Nervi, H. (2007). ¿Existe la Pedagogía?. Santiago: Editorial Universitaria.

OCDE. (2004). Informe de la Educación en Chile. Santiago: MINEDUC/OCDE.

OCDE. (2010). Educating Teachers for Diversity: Meeting the Challenge. París: OCDE Publishing.

ORIEAL. (2003). La privatización de la educación en Chile. Costa Rica: Oficina Regional de la Internacional de la Educación para América Latina.

Ortúzar, M., Flores, C., Milesi, C. y Cox, C. (2009). Aspectos de la formación inicial docente y su influencia en el rendimiento académico de los alumnos. En Camino al Bicentenario. Propuestas para Chile. (pp. 155-186). Santiago: Ediciones Universidad Católica.

Paredes, R. y Pinto, J.I. (2009). ¿El fin de la educación pública en Chile? Estudios de Economía, vol.36, n.1, 47-66.

Kuhn, T. (1980). La estructura de las revoluciones científicas. México: Fondo de Cultura Económica.

Redon, S., Toledo, L. y Rubio, G. (2009). La vivencia de "lo común" en la escuela: Prácticas y configuraciones de representación simbólica de cohesión social y construcción de comunidad en niños y niñas de cuatro a diez años de edad. Informe de investigación Proyecto FONIDE. Recuperado desde http://www.fonide.cl/DecPublico/documentos

Restrepo, B. (2004). La investigación-acción educativa y la construcción de saber pedagógico. Educación y Educadores, vol.17, 45-55.

Sánchez, M. (2003). Diversificación curricular y Diseño de práctica de aula intercultural en Educación Primaria: Posibilidades y Desafíos. Temuco: Instituto de Estudios Indígenas.

Schiefelbein, E. (1993). En busca de la escuela del siglo XXI. Santiago: Unesco/Unicef.

Sen, A. (2007). Primero la gente: una mirada desde la ética del desarrollo a los principales problemas del mundo globalizado. Barcelona: Deusto.

SITEAL. (2011). Cobertura relativa de la educación pública y privada en América Latina. Sistema de Información de Tendencia Educativa en América Latina. Septiembre 2011. Recuperado desde www.cedus.cl

Suárez, M. (2006). EL saber pedagógico de los profesores de la Universidad de Los Andes Táchira y sus implicaciones en la enseñanza (Tesis doctoral). Universidad Rovira I Virgili, TarragonaCataluña, España. 
Tardiff, M. (2005). Los Saberes del Docente y su Desarrollo Profesional. Madrid: Narcea

UNESCO. (2000). Primer Estudio Internacional Comparativo sobre Lenguaje, Matemática y factores asociados para alumnos del tercer y cuarto grado de la educación básica. Santiago: UNESCO/OREALC.

UNESCO. (2008). Primer Reporte. Segundo Estudio Regional Comparativo y Explicativo. Los aprendizajes de los estudiantes de América Latina y el Caribe. Santiago: UNESCO /OREALC.

UNICEF. (2011). La Voz de los Niños, Niñas y Adolescentes y Discriminación. Santiago: Unicef. Recuperado desde http://www.unicef.cl/pdf/PPTLaVozDiscriminacion2011.pdf

Vaillant, D. (2004). Construcción de la profesión docente en América Latina. Tendencias, temas $y$ debates ( $2^{\mathrm{a}}$ parte). Santiago: PREAL.

Valenzuela, I. (2006). Hacia la construcción del saber especializado del profesor: el saber pedagógico (Tesis para optar al grado de Magíster en Educación). Instituto de Educación, Pontificia Universidad Católica de Valparaíso.

Villalobos, C. y Valenzuela, J. (2012). Polarización y cohesión social del sistema escolar chileno. Ponencia presentada al Segundo Congreso Interdisciplinario de Investigación en Educación. CIIE 2012. Recuperado desde http://www.ciie2012.cl/?page=view_programa_completo

Villata, M. (2004). Construcción de la práctica pedagógica del docente en la sala de clase. Investigaciones en Educación, vol.6, n.1, 32-47.

Von Bertalanffy, L. (1974). Robots, hombres y mentes. Madrid: Guadarrama.

Zapata, V. (2005). La evolución del concepto 'saber pedagógico': su ruta de transformación. Educación y Pedagogía, vol.15, n.37, 175-184. 